doi:10.17659/01.2019.0052

Journal of Case Reports 2019;9(3):198-200

\title{
Bilateral Anterior Glenohumeral Dislocation after Epileptic Seizure
}

\author{
Ghassen Drissi, Maher Arfa, Khaked Mohamed, Nada Ben Brahim \\ Department of Orthopaedics, University Faculty of Medicine of Tunis, la Rabta Hospital, Tunis, Tunisia.
}

\section{Corresponding Author: \\ Dr. Ghassen Drissi \\ Email: drissi.ghassen@yahoo.fr}

This is an Open Access article distributed under the terms of the Creative Commons Attribution License (creativecommons.org/ licenses/by/3.0).

Received Accepted

Published

November 6, 2018

June 23, 2019

September 25, 2019

\begin{abstract}
Background: The unilateral glenohumeral dislocation is the most frequent form of joint dislocation, but the bilateral dislocation is very uncommon. Although the expected form of dislocation after an epileptic seizure is the posterior form, the bilateral anterior form is much rarer. Case Report: A 30 year old man presented to emergency department with pain and deformity of both shoulders following fall due to convulsive seizure. On physical examination at the emergency, we noticed a total loss of function of both upper limbs fixed in abduction that was irreducible. Standard plain X-ray assessment of both shoulders confirmed the diagnosis of bilateral anterior-internal dislocation. Conclusion: In unilateral shoulder dislocations, the incidence of posterior dislocations is markedly less than that of anterior shoulder dislocations. The opposite is true of bilateral shoulder dislocations. Bilateral anterior shoulder dislocations after an epileptic fit is very uncommon.
\end{abstract}

Keywords: Epilepsy, Joint Dislocations, Pain, Seizures, Shoulder Dislocation.

\section{Introduction}

The unilateral glenohumeral dislocation is the most common form of joint dislocation and the most frequent reason for consultation, the bilateral form is very uncommon [1]. Bilateral dislocation of the shoulder which is uncommon, is often associated with epileptic crises or electrocution and is the most common cause of posterior bilateral dislocations, while bilateral anterior dislocations are most often associated with trauma [2]. Bilateral anterior dislocations secondary to convulsions are very rare [3]. The following case report demonstrates a bilateral anterior shoulder dislocation as a result of epileptic fit.

\section{Case Report}

We relate a case of a 30 year old man who presented to emergency department with pain and deformity of both the shoulders following fall due to convulsive seizure. Patient was diagnosed as being a case of grand mal epilepsy and was on regular treatment. On physical examination at the emergency, we noticed a total loss of function of both upper limbs fixed in abduction that was irreducible. In addition, we noticed the "epaulette sign" and proeminence of the acromion [Fig.1-3]. No neurovascular abnormalities in the upper limbs were reported. Standard plain X-rays assessment of both shoulders confirmed the diagnosis of bilateral anterior-internal dislocation and no fractures were noted [Fig.4-6]. The dislocations were reduced easily and uneventfully employing the Milch technique under analgesia and sedation.

An examination looking for a sensitive deficit was performed after the reduction. X-rays were done to confirm that the dislocation was well reduced and to eliminate a fracture [Fig.7-8]. An immobilization was made for three weeks. Subsequently, progressive mobilization was started with pendulum exercises followed by muscle strengthening at three weeks. At followup the patient reported no pain and had returned to 
his normal activities of daily living with minimal discomfort.

\section{Discussion}

Bilateral shoulder dislocations are uncommon and almost always occur in the posterior direction. In 1902, bilateral shoulder dislocation was first found in patients in whom inordinate muscular contractions occurred as a result of Camphor overdose [4]. Bilateral anterior dislocation of the shoulder is an uncommon entity and few cases are found in the literature.

The mechanism of posterior dislocation is that the internal rotators of the humerus (latissimus dorsi, pectoralis major and subscapularis) which are more powerful than the external rotators (infraspinatous and teres minor) cause an excessive adduction and internal rotation during the seizure which results in a posterior dislocation of the humeral head [5]. Therefore, trauma is a more usual cause than seizures during bilateral anterior dislocation [6]. Many authors have suggested that bilateral anterior dislocation after an epileptic seizure cannot occur during muscular contractions, but following trauma of the shoulders striking the ground after the fall $[7,8]$. The care is the same as for unilateral dislocations [9]. Early reduction and immobilization should be ensued by final treatment, which may contain active and passive physiotherapy or surgery in the younger, more active patient group.

There is still no consensus regarding the protocol for actions after release from the emergency services. However, many authors have agreed on immobilization in internal rotation and adduction during three weeks followed by active mobilization with progressive pendular movements, plus physiotherapy [4].

\section{Conclusion}

Bilateral asymmetric dislocation of shoulders following an epileptic fit represents an emergency

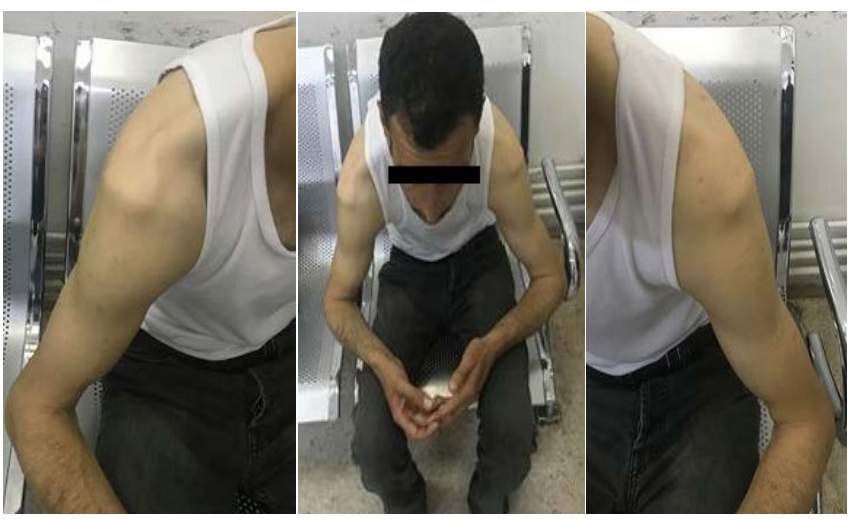

Fig.1-3: The appearance of both dislocated shoulders just before reduction maneuvers.

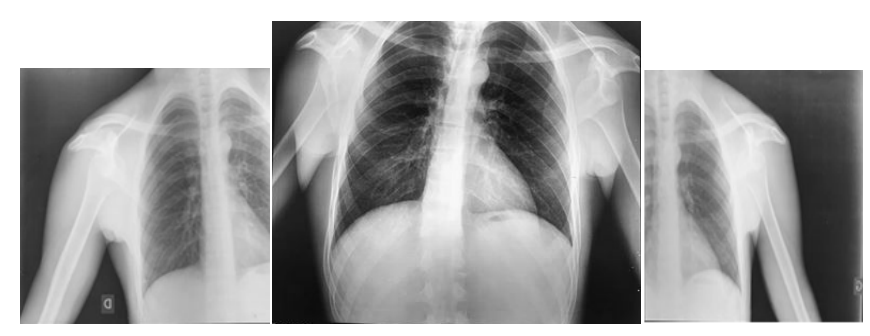

Fig.4-6: X-rays showing simultaneous recent anterior internal bilateral dislocation.
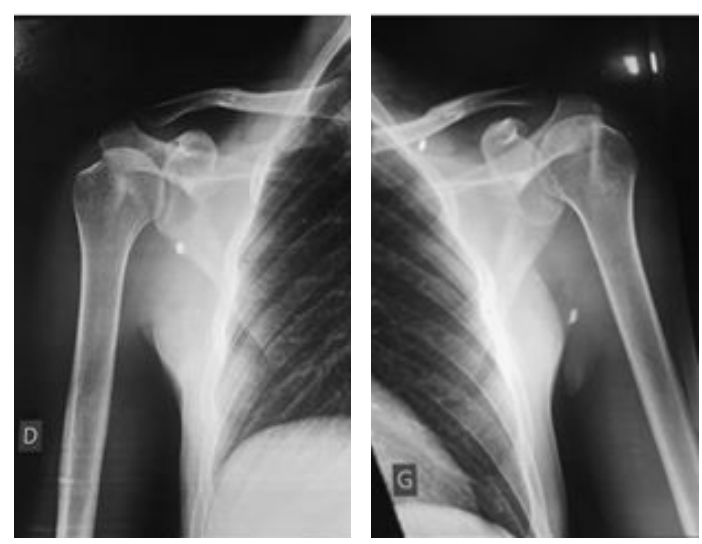

Fig.7,8: The anteroposterior radiographs of the two shoulders confirm the anatomical reduction.

imploring a quick diagnosis and reduction. The dislocation must be reduced in emergency to reduce pain and to avoid long-term complications like necrosis of the humeral head. The multidisciplinary approach to such patients can give good results. The possibility of shoulder instability must be kept in mind and need for regular follow-up. 
Contributors: DG: manuscript writing, patient management; AM, MK: manuscript editing, patient management; $\mathrm{BN}$ : critical inputs into the manuscript. DG will act as a study guarantor. All authors approved the final version of this manuscript.

Funding: None; Competing interests: None stated.

\section{References}

1. Suryavanshi A, Mittal A, Dongre S, Kashyap N. Bilateral anterior shoulder dislocation with symmetrical greater tuberosity fracture following seizure. J Orthop Case Rep. 2012;2(1):28-31.

2. Betz ME, Traub SJ. Bilateral posterior shoulder dislocations following seizure. Intern Emerg Med. 2007;2(1):63-65.

3. Maliyappa C, Al-Marboi BS, George M. Bilateral recurrent anterior fracture dislocation of shoulder joint due to grand mal epileptic convulsions. J Orthop Allied Sci. 2013;1:44.
4. Silva LP, Sousa CV, Rodrigues E, Alpoim B, Leal M. Bilateral anterior glenohumeral dislocation: clinical case. Revista Brasileira de Ortopedia (English Edition). 2011;46:318-320.

5. Wheelton A, Dowen D. A case of bilateral anterior gleno-humeral dislocation following first time seizure. J Orthop Case Rep. 2015;5(2):38-40.

6. Siu YC, Lui TH. Bilateral anterior shoulder dislocation. Arch Trauma Res. 2014;3:e18178.

7. Farikou I, Leroy GM, Daniel HE, Theophil C, Aurelien SM. Recurrent bilateral anterior simultaneous dislocation of the shoulder following epileptic seizures: A rare lesional association. Int J Ortho Res Ther. 2017;1:4.

8. Lasanianos N, Mouzopoulos G. An undiagnosed bilateral anterior shoulder dislocation after a seizure: a case report. Cases J. 2008;1:342.

9. Dunlop CCR. Bilateral anterior shoulder dislocation A case report and review of the literature. Acta Orthop Belg. 2002;68:3. 